PANORAMA

ISSN: $1909-7433$

ISSN: 2145-308X

ednorman@poligran.edu.co

Politécnico Grancolombiano

Colombia

\title{
LAS MÚSICAS COLOMBIANAS EN EL CURRÍCULO ADICIONAL DE LOS PROGRAMAS PROFESIONALES DE EDUCACIÓN MUSICAL DE IBAGUÉ
}

Salinas-Arias, Boris Alfonso

LAS MÚSICAS COLOMBIANAS EN EL CURRÍCULO ADICIONAL DE LOS PROGRAMAS PROFESIONALES DE EDUCACIÓN MUSICAL DE IBAGUE

PANORAMA, vol. 14, núm. 27, 2020

Politécnico Grancolombiano, Colombia

Disponible en: http://www.redalyc.org/articulo.oa?id=343964051003

DOI: https://doi.org/10.15765/pnrm.v14i27.1521

https://journal.poligran.edu.co/index.php/panorama/about/submissions\#copyrightNotice

Esta obra está bajo una Licencia Creative Commons Atribución-NoComercial-SinDerivar 4.0 Internacional 
Artículos de investigación científica y tecnológica

\title{
LAS MÚSICAS COLOMBIANAS EN EL CURRÍCULO ADICIONAL DE LOS PROGRAMAS PROFESIONALES DE EDUCACIÓN MUSICAL DE IBAGUÉ
}

\author{
COLOMBIAN MUSIC IN THE ADDITIONAL \\ CURRICULUM OF THE PROFESSIONAL MUSIC \\ EDUCATION PROGRAMS OF IBAGUÉ \\ A MÚSICA COLOMBIANA NO CURRÍCULO \\ ADICIONAL DOS PROGRAMAS PROFISSIONAIS DE \\ EDUCAÇÃO MUSICAL DE IBAGUÉ
}

Boris Alfonso Salinas-Arias boris.salinasarias@gmail.com Universidad del Tolima., Colombia

PANORAMA, vol. 14, núm. 27, 2020

Politécnico Grancolombiano, Colombia

Recepción: 21 Marzo 2020 Aprobación: 29 Julio 2020

DOI: https://doi.org/10.15765/ pnrm.v14i27.1521

Redalyc: http://www.redalyc.org/ articulo.oa?id=343964051003

https://journal.poligran.edu.co/ index.php/panorama/about/ submissions\# copyrightNotice CC BY-NC-ND
Resumen: El presente artículo es un estudio de caso con una posición teórica decolonial, cuyo objetivo fue revisar la presencia de las músicas colombianas en el currículo adicional de los programas profesionales de educación musical de la ciudad de Ibagué, los cuales son ofertados por el Conservatorio del Tolima. En primera instancia, el estudio buscó identificar el currículo adicional de la institución, encontrando que el principal espacio curricular complementario es el concierto, el cual se da en un importante salón que encaja en la definición de currículo como lugar. En segunda instancia, se encontró que la música clásica domina el salón de conciertos, en el cual la presencia de músicas colombianas es reducida. Los hallazgos del estudio muestran que las músicas colombianas presentes en este currículo adicional obedecen a los patrones de blanqueamiento propios de la colonialidad.

Palabras clave: Colonialidad, músicas colombianas, currículo adicional, currículo como lugar.

Abstract: This article is a case study with a decolonial theoretical position whose objective was to review the presence of Colombian music in the additional curriculum of the professional music education programs of the city of Ibagué, which are offered by the Conservatory of Tolima. In the first instance the study sought to identify the additional curriculum of the institution, finding that the main complementary curricular space is the concert, which takes place in an important hall that fits the definition of curriculum as a place. In the second instance, it was found that classical music dominates the concert hall in which the presence of Colombian music is reduced. The findings of the study show that the Colombian music present in this additional curriculum obeys the whitening patterns characteristic of the Coloniality.

Keywords: Coloniality, Colombian music, additional curriculum, curriculum as a place.

Resumo: O presente documento é um estudo de caso com uma posição decolonial, o objetivo foi fazer uma revisão sobre a presença das músicas de Colombia no currículo adicional dos programas profissionais de educação musical da cidade de Ibagué, estes são oferecidos pelo Conservatorio del Tolima. Primeiro, o estudo identifica o currículo adicional da instituição achando que o principal espaço adicional é o concerto, este é realizado em uma sala de concertos que se encaixa na definição de currículo como local. Segundo, foi encontrado que a música clássica tem uma posição dominante no 
currículo adicional, ao contrário da música de Colombia que tem uma presença reduzida. Os resultados mostram que a musica de Colombia presente neste curriculo adicional obedece aos padrões clareadores da colonialidade.

Palavras-chave: Colonialidade, música de Colombia, currículo adicional, currículo como local.

\section{INTRODUCCIÓN}

De manera tradicional, la educación musical profesional, no solo en la ciudad de Ibagué sino en Colombia, se ha basado en el estudio de la música europea, relegando a un plano de exclusión a las músicas colombianas (Muñoz, 2015). Este fenómeno ha sido debatido desde la educación musical por autores como Ayala, (2013); Ochoa (2011); Gallardo (2016), y desde la propuesta para la enseñanza de la gramática musical con base en melodías de música tradicional colombiana, con base en el marco teórico del proyecto Modernidad/Colonialidad, al cual pertenecen autores como Aníbal Quijano, Maldonado Torres y Walter Mignolo.

El presente documento es un estudio enmarcado en un pensamiento decolonial, cuyo objetivo principal es identificar y analizar el currículo adicional de los programas de formación musical profesional de Ibagué, en el que están presentes las músicas colombianas, para poder establecer las características específicas de esa presencia sonora. Lo anterior, debido a que a la ciudad de Ibagué le acompaña la paradoja de que, siendo reconocida popularmente como la Ciudad Musical de Colombia, en su institución educativa musical insignia, el Conservatorio del Tolima, era prohibido el estudio de las músicas colombianas hasta la década de los 90 , época desde la cual estas músicas comienzan a ingresar paulatinamente a la institución (Arias, 2018).

Desde que comenzó el presente estudio se pudo identificar que los programas profesionales de educación musical de Ibagué son ofertados únicamente por el Conservatorio del Tolima ${ }^{[2]}$, institución centenaria que tiene una rica historia, pero que hasta los años 90 comenzó a ofertar su primer programa de educación musical profesional, denominado Licenciatura en Música, al cual se le sumó un segundo programa en el año 2007, llamado Maestro en Música (PEI CT ${ }^{\text {[3] }}$, 2015). Sobre esta institución y estos programas se realizó la primera delimitación.

Para que el lector pueda comprender el panorama de la inclusión curricular de las músicas colombianas en los programas de educación musical profesional de Ibagué, se le sugiere remitirse al artículo titulado 'El ingreso de las músicas colombianas a la educación superior del Conservatorio del Tolima' (Arias, 2018).

\section{MÉTODO}

Este trabajo hace parte del proyecto Las músicas colombianas en los programas de formación musical profesional de Ibagué: apuntes sobre currículo y colonialidad, el cual usó como estrategia de investigación el 
estudio de caso frente al fenómeno de la no inclusión de las músicas colombianas en la institución insignia de la Ciudad Musical de Colombia. El presente artículo se centra en identificar las músicas colombianas presentes en el currículo adicional.

Siguiendo a Posner (2005), el currículo oficial es "el que está descrito en los documentos oficiales" (p.15), y se diferencia del currículo adicional en que este último está compuesto por "las experiencias planeadas fuera del currículo formal" (p.15). El currículo adicional son todas aquellas actividades académicas que están por fuera de las clases y de la malla curricular, se caracteriza por la participación voluntaria del estudiante y por la no mediación de una nota o calificación que incida en el rendimiento académico del programa estudiado, también confirmado por el estudio de Castro et al., (2014), Inclusión de la educación artística en el currículo de formación profesional.

Este artículo presenta el análisis realizado a espacios curriculares adicionales -o complementarios- que por su naturaleza tienen poca visibilidad en el currículo oficial. Para ello lo primero que se realizó fue la identificación de esos espacios, con lo cual se encontró que en el Conservatorio del Tolima se oferta principalmente una temporada anual de conciertos concertada con el Ministerio de Cultura de Colombia, así como un Festival Internacional de piano y otros eventos como talleres y encuentros a los cuales la institución es invitada. Dada la particularidad de la programación encontrada en la institución, se analizó el periodo 2015, esto es, todos los eventos realizados durante ese año.

Luego de identificar los espacios curriculares adicionales se hizo una recolección y revisión documental de los programas de mano de eventosrecitales, conciertos y talleres- los cuales son la fuente primaria de información. En estos programas se encuentra los datos discursivos que permiten conocer cuáles son las músicas colombianas que se interpretan, en qué formatos, a qué regiones pertenecen y qué música domina el panorama escénico en la institución.

Los programas de mano se obtuvieron de manera física y en formato digital, facilitados por la Oficina de archivo y la Coordinación académica de la institución. Luego se organizó la información de forma inicial en diversas categorías y posteriormente se usó la técnica de análisis e interpretación de la información para el estudio de caso, propuesta por Stake (1999), la cual se presenta en tres procedimientos: (i). Suma categórica o interpretación directa; (ii). Correspondencia o modelos; y (iii). Generalizaciones naturalistas. A continuación, se desglosan estos tres procesos.

- La suma categórica o interpretación directa: son dos maneras de dar significado a los casos; la suma categórica consiste en realizar una suma de la información para poder organizarla, ya que hay datos o ejemplos que al ser tomados individualmente pueden carecer de significado; en este caso el investigador se percata de la existencia de datos aparentemente aislados que están relacionados con otros, por lo cual realiza una suma por categorías correlacionando la información dándole un significado. La interpretación directa se realiza cuando hay datos o ejemplos individuales 
que ofrecen por sí mismos un significado. Estas dos formas de realizar el análisis son escogidas por la intuición del investigador al estar en contacto con la información durante y después del trabajo de campo, por tal motivo no se pueden anticipar, pues dependen de la interacción con el fenómeno estudiado.

- Correspondencia y modelos: la información recolectada se analiza buscando una correspondencia entre ella y modelos existentes que se encuentran en la literatura o marco teórico. Una vez realizada la suma categórica y la interpretación directa, se analiza la información y se busca correspondencia con modelos establecidos, que, para este escrito, son los fenómenos de la colonialidad y el eurocentrismo, sin descartar otros posibles modelos emergentes a los que puede orientar el estudio.

- Generalizaciones naturalistas: Stake (1999), señala que si bien el estudio de caso no es un estudio de muestreo por el cual se pueda crear teoría para generalizar sobre los fenómenos estudiados, este sí permite aprender de fenómenos que son generales y familiares para los lectores de la investigación, los cuales se proyectan como conclusiones y aprendizajes de vida producto del caso.

Las músicas colombianas fueron analizadas desde tres tópicos: (i) instrumentos musicales, (ii) formatos instrumentales y (iii) repertorios. A partir de estos se identifica el aire o estilo de música colombiana y/o lugar de procedencia, compositores y demás datos de interés. Para efectos de este artículo se entiende por músicas colombianas a los diversos géneros y estilos musicales que hacen parte del folclore nacional, incluyendo piezas folclóricas que han traspasado las barreras de la música popular, académica o comercial.

Finalmente, hay que aclarar que este trabajo no es un análisis cuantitativo de datos, aunque sí se encontraron algunos datos que pueden tener una visión cuantificable.

\section{DISCUSIÓN}

\section{El salón de conciertos Alberto Castilla: el currículo como lugar}

El primer factor que sale a luz al revisar el currículo adicional de los programas de educación musical profesional del Conservatorio del Tolima es el espacio físico en que este ocurre, El salón Alberto Castilla, construido en 1931 (PEI, 2015), que con la Ley 112 de 1994 pasó a ser -al igual que buena parte de las instalaciones de la institución- monumento nacional.

Allí -en el salón de conciertos- hay una serie de elementos importantes como el hecho de que este espacio físico no sea parte del currículo oficial, al no estar reflejado en la malla curricular, lo cual contrasta con que sea lo primero que impresiona al visitante al ingresar a la institución, por ser un sitio cargado de información histórica importante para la ciudad. Este es el principal escenario curricular adicional de aprendizaje musical del Conservatorio del Tolima. 
El Conservatorio del Tolima -incluyendo su salón de conciertos- encaja en lo que Pinar (2004) y Kincheloe (1991), llamaron el currículo como lugar. Para Kincheloe, "el lugar es lugar siempre que se vincule con una historia" (1991, p.8), y Pinar posteriormente lo define como "el concepto que entrelaza las particularidades de la historia, la cultura y la subjetividad" (2014, p.241). En consecuencia, el lugar es un espacio que permite reconstruir el pasado gracias a la existencia de vestigios vivos, a los que Kincheloe llama tesoros.

Pese a que Ibagué fue fundada en el año 1550, esta no posee un centro histórico y su patrimonio arquitectónico es escaso ${ }^{[4]}$. En medio de esa realidad, el salón Alberto Castilla es un vestigio vivo que representa no solo la arquitectura republicana de la ciudad, sino también el título de Ibagué, Ciudad Musical de Colombia.

El currículo como lugar se presenta acá por medio de este tesoro que es el salón Alberto Castilla y los abundantes detalles que lo acompañan, como los 16 óleos donde están retratados importantes músicos académicos o clásicos -música de Europa central de entre los siglos XVI y XX-, y su diseño para grupos de cámara.

Así mismo, este salón de conciertos está lleno de las historias de los diferentes artistas que han hecho música en su espacio y de otros sucesos que le dan sentido a este lugar ${ }^{[5]}$, fundiendo en cada concierto lo arquitectónico con el sonido musical, lo que da vida a una cultura erudita y académica de la música propia de los conservatorios europeos que sostienen una tradición sonora de varios siglos de música clásica y academicista. Este sitio es muestra viva de la hegemonía de la música clásica y, a su vez, de forma oculta -currículo oculto-, de exclusión de las músicas populares no europeas.

Este currículo como lugar es también un espacio curricular abierto a la ciudad y complementario para los estudiantes del Conservatorio del Tolima, permite tener una experiencia de aprendizaje sobre la música clásica y la cultura de la música académica. En él converge una gran cantidad de conciertos que se presentan como espacios prácticos de aprendizaje desde la audición musical, que no solo benefician a los estudiantes del Conservatorio del Tolima, sino que acoge al público general que ingresa de manera libre a eventos realizados durante todo el año. Allí la ciudadanía puede encontrar un tesoro vivo de la Ciudad Musical de Colombia.

\section{Los espacios curriculares adicionales.}

En este lugar -salón Alberto Castilla- ocurre el currículo adicional de los programas de educación superior del Conservatorio del Tolima, el cual se nutre de una cantidad importante de eventos artísticos realizados por la institución, que se agrupan en dos grandes momentos: (i) el IV Festival Internacional de Piano "Óscar Buenaventura", y (ii) la Temporada de Conciertos 2015. En estos eventos participaron los estudiantes como asistentes y/o intérpretes solistas o miembros de las 
agrupaciones musicales de acuerdo con la investigación de Carter (2019): "Perspectivas sobre la inclusión de la performance en la educación artística escolar secundaria en Latinoamérica".

El Festival Internacional de Piano que realiza el Conservatorio del Tolima tuvo su primera versión en el mes de mayo de 2009, y celebró en el 2017 su sexta edición. El evento lleva su nombre como homenaje póstumo al músico Óscar Buenaventura, quien perteneció a una familia con formación musical de la ciudad de Ibagué[6], fue estudiante del Conservatorio del Tolima, posteriormente continuó su formación musical en Estados Unidos y tuvo una notoria carrera a mediados del siglo XX. Sin embargo, pese a su trayectoria, este instrumentista ha caído paulatinamente en el olvido (Prudencio, 2004).

ElVI Festival Internacional de Piano Óscar Buenaventura fue realizado entre el 11 y el 15 de mayo del 2015; participaron veinticuatro intérpretes del piano, cuatro de ellos internacionales, diez nacionales y diez locales, que mostraron su trabajo en un total de once conciertos y una conferencia.

El segundo momento, el cual aporta el mayor número de eventos realizados en la institución, es la Temporada de Conciertos 2015, la cual es financiada principalmente por el Conservatorio del Tolima y apoyada por el Programa Nacional de Concertación Cultural del Ministerio de Cultura. Esta temporada aportó entre el 3 de marzo y el 4 de diciembre de 2015 la importante suma de 43 eventos, lo que equivale a un evento cada cuatro días. En esta temporada anual de conciertos participa la comunidad académica del Conservatorio del Tolima por medio de sus distintas agrupaciones, conformadas por estudiantes y docentes.

Además de los conciertos no se encontró otro espacio curricular adicional, lo que hace notable la falta de espacios de aprendizaje teóricos que potencien no solo el currículo como lugar, sino también que ofrezcan variedad, pues el concierto es por lejos el único espacio de aprendizaje existente. -en los programas de mano solo se encontró un foro y un taller-.

\section{RESULTADOS}

\section{Las músicas presentes en el currículo adicional}

\section{Música clásica: amplia dominadora del currículo adicional}

En las tablas 1 y 2 se presentan el número de eventos realizado durante el año 2015, siendo el cuadro 1 los eventos del VI Festival Internacional de Piano Óscar Buenaventura, y el cuadro 2 los eventos de la Temporada de Conciertos 2015. En estos cuadros aparece la fecha de cada concierto, la agrupación que se presentó en el evento, el número de obras colombianas que fueron interpretadas en comparación con las obras no colombianas, el continente y país de origen del repertorio - europeo, norteamericano, latinoamericano y colombiano, no se encontró repertorio africano ni asiático-. 


\begin{tabular}{llcc}
\hline $\mathbf{N}^{\circ}$ & \multicolumn{1}{c}{ Formato } & $\begin{array}{c}\text { Origen del } \\
\text { repertorio }\end{array}$ & $\begin{array}{c}\text { Obras colombianas vs obras } \\
\text { no colombianas }\end{array}$ \\
\hline $\mathbf{1}$ & Orquesta sinfónica, dos pianos y piano solista & Europeo & No obras col. \\
\hline $\mathbf{2}$ & Recital de piano solista & Europeo & No obras col. \\
\hline $\mathbf{3}$ & Recital piano solista & Europeo & No obras col. \\
\hline $\mathbf{4}$ & Recital piano solista & $\begin{array}{c}\text { Europeo y } \\
\text { colombiano }\end{array}$ & 1 col. vs 5 no col. \\
\hline $\mathbf{5}$ & $\begin{array}{l}\text { Conferencia "Relación entre la actitud postura y el desempeño frente al piano a } \\
\text { partir de la técnica Alexander" }\end{array}$ & No aplica & No aplica \\
\hline $\mathbf{6}$ & Recital piano solista & $\begin{array}{c}\text { Europeo y } \\
\text { latinoamericano }\end{array}$ & No obras col. \\
\hline $\mathbf{7}$ & Recital piano solista & Europeo & No obras col. \\
\hline $\mathbf{8}$ & Recital piano solista & $\begin{array}{c}\text { Europeo y } \\
\text { colombiano }\end{array}$ & 1 col. vs 11 no col. \\
\hline $\mathbf{9}$ & Recital piano solista & Europeo & No obras col. \\
\hline $\mathbf{1 0}$ & Recital piano solista & Europeo & No obras col. \\
\hline $\mathbf{1 1}$ & Recital piano solista & Europeo & No obras col. \\
\hline $\mathbf{1 2}$ & Orquesta sinfónica y piano & Europeo & No obras col. \\
\hline
\end{tabular}

Tabla 1

VI Festival internacional de Piano Óscar Buenaventura, 2015.

Fuente: Elaboración propia

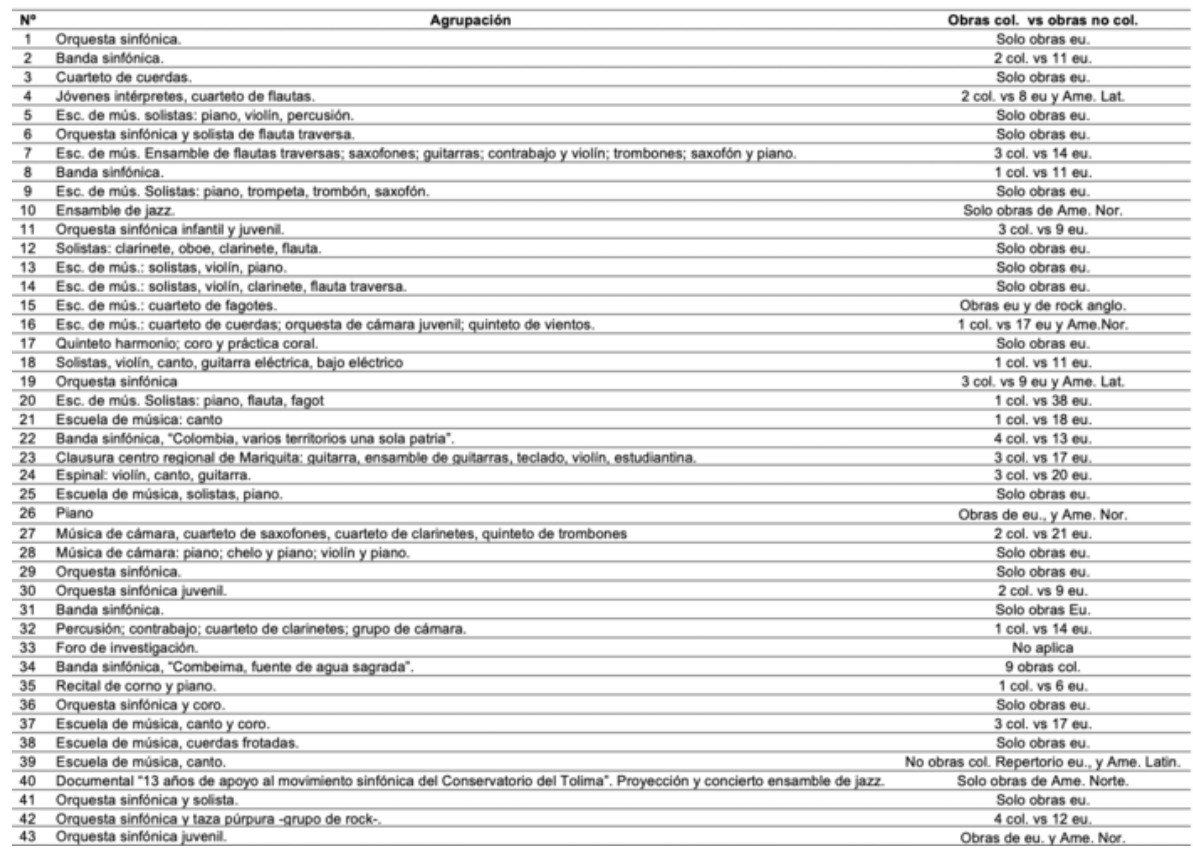

Tabla 2.

Temporadas de Conciertos 2015.

Fuente: Elaboración propia

En la Temporada de Conciertos de las 43 actividades, los conciertos 1, $3,5,6,9,12,13,14,17,25,28,29,31,36$, y 41 son exclusivamente de música europea, en total 15 que suman un poco más de la tercera parte de los eventos realizados, y solo en los conciertos $10,18,33$ y 40 no estuvo presente la música europea, cuatro eventos que entre 42 equivale a menos de una décima parte de los conciertos. En cuanto al Festival de Piano, en los 12 conciertos realizados estuvo presente la música europea. Aquí una primera conclusión, y es que la música europea domina el currículo adicional.

Por su parte, las músicas colombianas estuvieron presentes en 19 eventos de la Temporada de Conciertos - conciertos 2, 4, 7, 8, 11, 16, $18,19,21,22,23,24,27,30,32,34,35,37$ y 42, menos de la mitad, un poco más del 44\%-, y solo un evento, el número 34 , fue de manera 
exclusiva de músicas colombianas. Así mismo, puede verse que en todos los eventos en los que las músicas colombianas compartieron escenario siempre fueron la cuota minoritaria del programa, llegado incluso a tener únicamente una obra colombiana frente a 11 y en algunos casos 18 obras extranjeras. Por su parte, en el Festival de Piano, las músicas colombianas estuvieron presentes en dos de doce eventos -concierto 4 y 10-, con dos piezas cortas.

Sin ser este un estudio estadístico, salta a la vista que las músicas colombianas representan la cuota menor en los conciertos realizados durante el año 2015, incluso la música de Norteamérica tuvo dos conciertos exclusivos para su repertorio en la Temporada Anual de Conciertos (10 y 40), frente a un evento exclusivo para el repertorio colombiano.

\section{Los instrumentos y formatos musicales}

$\mathrm{Al}$ revisar los formatos musicales -e instrumentos- en los cuales se interpretan las músicas colombianas, solo en el evento número 23 de la Temporada de Conciertos 2015 aparece una estudiantina, agrupación de la Escuela de Música y única de músicas colombianas, ya que en los demás conciertos los formatos presentes son orquestas sinfónicas, tanto principal como infantil y juvenil, banda sinfónica, cuartetos de flautas, trombones, instrumentos solistas -con acompañamiento de piano- como trompetas, clarinetes, saxofones, voz. También hubo agrupaciones como orquestas y grupos de cámara, coros, ensamble de guitarras y conjunto de jazz. Ospina (2015), lo ratifica en su artículo titulado "Panorama sobre el conocimiento construido acerca del jazz en los trabajos de grado de la Licenciatura en Música de la Universidad Pedagógica Nacional".

Aunque en la Temporada de Conciertos 2015 los formatos instrumentales de músicas colombianas son casi inexistentes, a no ser por la estudiantina -que pertenece a la Escuela de Música[7]-. Empero, pese a que solo existe una agrupación de músicas colombianas, es de resaltar que los conjuntos de música clásica, como la orquesta sinfónica, hicieron el montaje de repertorios colombianos, permitiendo espacios para poder encarar estas músicas de manera práctica.

La banda sinfónica incluyó en cuatro de sus presentaciones en la Temporada de Conciertos a las músicas colombianas (eventos 2, 8, 22 y 31), así mismo, las orquestas sinfónica institucional, juvenil e infantil (eventos 11, 19, 30 y 42), y los estudiantes de canto y coro (eventos 18, 21, 24 y 37); los ensambles de cámara como el cuarteto de flautas, el ensamble de guitarras, la orquesta de cámara infantil y el cuarteto de clarinetes incluyeron las músicas colombianas en cinco eventos $(4,7,16$, 27 y 32). Por su parte, los intérpretes solistas de saxofón, piano y guitarra incluyeron las músicas colombianas en tres eventos (7, 20 y 24).

Lo anterior deja ver que de manera significativa los diferentes directores de las agrupaciones, así como los estudiantes en sus trabajos solistas se interesan por incorporar músicas colombianas en sus presentaciones, situación que contrasta con el hecho de que solo haya un único formato 
de músicas colombianas, la estudiantina, la cual realizó una presentación en todo el año (evento 23).

\section{El repertorio}

En la tabla 3, se organizó las distintas piezas colombianas interpretadas en la Temporada de Conciertos 2015 y el VI Festival Internacional de Piano, presentando el nombre de la composición, autor, ritmo y zona geográfica de procedencia. En el año 2015 fueron interpretadas 32 obras colombianas de un total de 25 compositores; algunas de estas obras se interpretaron hasta en tres ocasiones - en diferentes fechas, cabe aclarar-. En total, las músicas colombianas sonaron 41 veces.

\begin{tabular}{|c|c|c|c|}
\hline Ritmo & Pieza & Autor & Region \\
\hline Bambuco & Muchacha de risa loca & Lucho Ramirez & Andina \\
\hline Bambuco & Gloria Beatriz & León Cardona & Andina \\
\hline Bambuco & La guanenta & Nicanor Dlaz & Andina \\
\hline Bambuco & Pequenta sulto. I. Bambuco & Adollo Mejia & Andina \\
\hline Bambuco & El republicano & Luis A. Calvo & Andina \\
\hline Bambuco & Combeima, historia de un bambuco en cuatro estancias & Andrés Mauricio Acosta & Andina \\
\hline Bambuco & El tolimense & Gentll Montaña, Noel Charry & Andina \\
\hline Bambuco & Rapsodia bambuco & Gabriel Solo (estudiante) & Andina \\
\hline Bambuco & EI navegante & J. de la Pava & Andina \\
\hline Pasillo & Rio Call & Sebastán Solari & Andina \\
\hline Pasillo & Michel & Genta Montana & Andina \\
\hline Pasillo & Aires de mi tierra & Gustavo Gomez & Andina \\
\hline Pasillo & Humorismo & Avaro Romero & Andina \\
\hline Pasillo & Chaflan & D.R.A & Andina \\
\hline Pasillo & Edelma & Terig Tued & Andina \\
\hline Torbellino & Color torbellino & Andrés Acosta & Andina \\
\hline Guabina & Viviras mi Tolima & Pedro J. Ramos & Andina \\
\hline Bunde - Guabina- & Noches del Tolima & José Maria Tena & Andina \\
\hline Torbellino & Pequefia sulte. II. Torbellino y canción & Adollo Mejia & Andina \\
\hline Cumbia & Pequefia sulte. III. Cumbia & Adollo Mejia & C. Altantice \\
\hline Cumbia & Yo me tamo Cumbia & José Bamos & C. Aläntica \\
\hline Cumbia & Cumbiamba & Maunicio Murcia & C. Allantica \\
\hline Cumbia & Tolú; Colombia tierra querida & Lucho Bermúdez & C. Allantica \\
\hline Porro & Pachino ecthe & Alex Tovar & C. Alantica \\
\hline Porro & Caprichito & Lucho Bermúdez. & C. Alántice \\
\hline Vallenato & La cantera; La foto de los dos & Cartos Vives & C. Alantice \\
\hline Mapalte & El mapale & Camilo Piñeres & C. Allantice \\
\hline Mapalt & Prende la vela & Lucho Bermǘdez & C. Alántica \\
\hline Currulao & Mi Buenaventura & Petronio Ávarez & C. Pacifica \\
\hline Callipso & Calipso & Jessis Alberto Rey & Islefía \\
\hline
\end{tabular}

Tabla 3.

Músicas colombianas y compositores presentes en el currículo adicional, 2015 Fuente: Elaboración propia

El repertorio colombiano interpretado en el año 2015 es dominado por la música andina colombiana, exclusivamente por los bambucos y pasillos, dejando en un segundo plano las guabinas y torbellinos. No aparecen rumbas criollas, merengues campesinos, cañas, etc. La música andina colombiana se ve reducida y representada en esos cuatro aires.

Después aparece la música colombiana de la zona Atlántica, con dominio de la cumbia y menor presencia del vallenato, el porro y el mapalé; no aparecen la puya, la gaita, la tambora, ni otros ritmos de esta zona; en cuanto al Pacífico, el único aire presente es el currulao, con el himno popular Mi Buenaventura; y en último lugar aparece un único ritmo de música isleña, el calipso.

En total solo hubo diez ritmos colombianos de toda la inmensidad sonora de la nación que se hicieron presentes en el salón Alberto Castilla, 
no hubo música de los indígenas, ni música de Nariño o los Llanos Orientales.

Respecto a los compositores de las obras, algunos son destacados músicos académicos como Gentil Montaña, otros, músicos de importante arraigo académico y popular como Luis A. Calvo, y otros, músicos que tuvieron un importante impacto comercial como Lucho Bermúdez, José Barros y Carlos Vives.

Entre las 32 obras colombianas interpretadas hay dos que son autoría de un docente de la institución, el señor Andrés Mauricio Acosta, -Color torbellino, evento número 11, interpretada por la Orquesta Sinfónica Juvenil; y Combeima, Historia de un bambuco en cuatro estancias, evento 34, interpretado por la Banda Sinfónica-. Igualmente, se interpretó una obra colombiana compuesta por un estudiante del Conservatorio, Gabriel Soto -Rapsodia bambuco, evento 34, obra para corno y piano-.

Estos datos muestran que las músicas colombianas son de interés para docentes y estudiantes de la institución, por lo menos desde el punto creativo, pero aún está lejos de ser una misión institucional, de lo contrario habría un mayor esfuerzo por propiciar la creación de agrupaciones de músicas colombianas, el montaje, arreglo, composición, puesta en escena de estas músicas y más aún, espacios de reflexión y diálogo sobre estas músicas.

\section{REFLEXIONES FINALES}

Los resultados del estudio develan que la música clásica domina el espacio curricular complementario en el cual las músicas colombianas tienen una pequeña participación. Esta hegemonía de la música clásica se ve en los formatos y el repertorio interpretado, pero destaca el hecho de que estos mismos formatos interpreten músicas colombianas y que en la programación haya presencia de dos obras colombianas compuestas por miembros de la comunidad académica del Conservatorio del Tolima, lo que demuestra interés de algunos estudiantes y docentes por estas músicas.

El estudio arroja igualmente que las músicas populares y de Norteamérica tienen una mayor presencia que las músicas colombianas, aunque esa presencia no sea igual a la que tiene la música clásica.

En cuanto a los formatos y el tipo de músicas colombianas presentes en los espacios curriculares complementarios, se logra identificar una hegemonía de la música andina colombiana, seguida de la música de la zona Atlántica del país, con una pequeña participación de la música del Pacifico y de la zona isleña. Las músicas de los Llanos Orientales y de las comunidades indígenas no están presentes.

Lo encontrado concuerda con lo propuesto en el marco de los estudios coloniales, y es que los presaberes pertenecientes a las comunidades racializadas se van insertando en el saber a medida que se blanquean, al mezclarse con la música clásica o academicista. Efectivamente, el grado de inclusión de las músicas colombianas en este espacio curricular complementario está relacionado con el nivel de academicismo y blanqueamiento de estas músicas, ya que son las músicas andinas 
colombianas las que han vivido en mayor medida el proceso de blanqueamiento, seguidas por las músicas de la zona Atlántica, que adicionalmente han tenido un importante auge comercial desde los años cincuenta.

Por otro lado, las músicas con menor proceso de blanqueamiento, como las de los pueblos indígenas, aún no ingresan a la sala de conciertos del Conservatorio del Tolima. Este hecho es más notorio cuando se piensa desde los formatos musicales, los cuales son mayoritariamente de música clásica y presenta un único formato de música colombiana, la Estudiantina, un conjunto híbrido mezcla de las estudiantinas de origen español conjugado con instrumentos mestizos colombianos como el tiple, propuesto por Morales Pino a finales del siglo XIX[8], el cual ayudó en el proceso de blanqueamiento de la música andina colombiana -sus ritmos, músicos e instrumentos musicales- sacándolos de su estigma de inferioridad.

Se puede apreciar que parte de la inclusión de las músicas colombianas en el currículo complementario se debe al interés de los estudiantes y algunos docentes, pero es claro que falta una mayor política de inclusión por parte de la institución, esto es, tomar decisiones estructurales como la creación de ensambles, realización de eventos académicos -congresos, coloquios, conferencias de música-, así como eventos musicales donde se promueva el diálogo, estudio y comprensión de las músicas colombianas.

No es el objetivo de este artículo debatir sobre si los conservatorios deban redefinir o no su perfil musical, pero sí llama la atención que el Conservatorio del Tolima se encuentre en una especie de encrucijada: por un lado, el ser una institución conservadora de la música europea $y$, por el otro, el ser una universidad musical que prepara docentes e instrumentistas que salen a trabajar en un campo laboral dominado por las músicas populares.

Estudios como el de Green (2003), y Zuleta y Jaramillo (2003), muestran que en el mercado mundial y colombiano de la música clásica representa rangos menores al $4 \%$, frente a las músicas populares y locales que llega a cifras que van del $58 \%$ al $95 \%$, lo cual se suma a la desaparición de orquestas sinfónicas que se está viviendo en el país, [9] y la baja audiencia que tiene esta música. Frente a esta realidad, el Conservatorio del Tolima se levanta como defensor del movimiento sinfónico y materializa esta lucha con su temporada de conciertos y el Festival de Piano, que le aportaron a la ciudad de Ibagué 53 conciertos y recitales en el año 2015, eventos que año a año son realizados por la institución.

El dilema de la universidad parece ser: modernización vs tradición. Los conservatorios son de los pocos lugares donde se mantiene viva la música clásica, pero a su vez forman músicos a los cuales excluye del estudio de la música popular y de las músicas nacionales. Parece ser que el conocimiento que adquieren los músicos profesionales sobre la música de su país se da más desde un currículo paralelo, que ocurre en la práctica cotidiana de hacer música con otros músicos en espacios no formales de educación valdría la pena hacer un estudio comparado al respecto-. 
Esto último se funde con un elemento: pensar el currículo como lugar desde las músicas colombianas y nacionales ¿Cuál es el lugar al cual pertenecen y en el que se hacen las músicas colombianas? ¿Debería la universidad musical pensar currículos como lugares acordes con los conocimientos que se quieren enseñar?

Durante el VI Festival Internacional de Piano Óscar Buenaventura ocurrió un hecho curioso que vale la pena mencionar. El concierto principal del evento tenía como protagonista a un músico cubano y el repertorio que interpretó fue completamente europeo. Sin embargo, al terminar el concierto, todo el público aplaudió con fervor pidiendo de común acuerdo, pero sin decir palabras, que por favor interpretara música de su tierra natal, música cubana. Efectivamente, el intérprete volvió al piano para tocar la música de su país durante media hora (diario de campo del autor, 2015).¿Por qué la programación del evento realizado en el salón de conciertos de música clásica no incluyó desde el principio este recital de piezas cubanas?

Vale la pena preguntar si un currículo como lugar anula de cierta manera otras prácticas culturales, como que las músicas populares son mejor vistas en exteriores y escenarios abiertos, y no tanto en auditorios de música clásica, elemento que excede este escrito, pero queda como pregunta de investigación.

\section{CONCLUSIÓN}

Finalmente, llama la atención la doble naturaleza curricular del concierto. Por un lado, el participar del conjunto musical hace parte del currículo oficial en cuanto a que aparece gestionado como un ensamble en la malla curricular, por lo cual el estudiante en algún momento de su carrera profesional tendrá que integrar alguna agrupación. Pero, por otro lado, el concierto es inequívocamente parte del currículo adicional en cuanto a que los estudiantes y la ciudadanía en general puede asistir a estos eventos como público, sumado a que todo estudiante de manera voluntaria también puede integrar las agrupaciones sin la obligación de tener que estar matriculado en la asignatura de Conjunto -como aparece en la malla curricular-.

Esta doble naturaleza curricular del concierto hace pensar en lo positivo que puede ser gestionar el currículo de manera que le permita a la universidad una mayor proyección social, elemento clave que en el caso de este Conservatorio le permite tener una temporada de conciertos que año a año va en crecimiento, llegando a la suma de un concierto cada tres días, elemento que sin lugar a duda cumple un rol social de importancia para la ciudad. Esto ahorrando en gastos, pues se tiene agrupaciones musicales de buen nivel con músicos que están en formación y, a su vez, benefician a la institución, a los otros estudiantes y a la comunidad en general. 


\section{REFERENCIAS}

Arias, B. (2018). El ingreso de las músicas colombianas a la educación superior del Conservatorio del Tolima. Revista Electrónica de LEEME, (41).

Asencio, R. (2013) Las Estudiantinas del antiguo Carnaval Alicantino: origen, contenido lírico y actividad beneficia (1860-1936). Salamanca, España: Universidad de Alicante. Editorial Agua Clara, SL.

Carter, R. (2019). Perspectivas sobre la inclusión de la performance en la educación artística escolar secundaria en Latinoamérica. Palobra: Palabra que obra, 19(2), 162-172.

Castro, M., Reyes, F., \& Cardona, C. (2014). Inclusión de la educación artística en el currículo de formación profesional. Praxis, 10(1), 176-188.

Gallardo, I. (2016). Propuesta para la enseñanza de la gramática musical con base en melodias de música tradicional colombiana. Trabajo de grado, Licenciatura en Música. Universidad Pedagógica Nacional.

González, J. (2006). "No doy por todos ellos el aire de mi lugar": la construcción de una identidad colombiana a través del bambuco en el siglo XIX. Tesis doctoral. Universidad Autónoma de Barcelona. Barcelona, España.

Ayala, C. (2013). ¿Qué música está al interior del departamento de Música de la Universidad del Cauca? Tesis de Maestría. Universidad de San Buenaventura. Popayán, Colombia.

Conservatorio del Tolima. (2015). Programación, IV festival internacional de Piano Oscar Buenaventura. Ibagué, Colombia: Facultad de Educación y Artes, Conservatorio del Tolima.

Conservatorio del Tolima (2015). Proyecto Educativo Institucional (PEI). Ibagué, Colombia: Facultad de Educación y Artes, Conservatorio del Tolima.

Demmer, F. (2011). Orquestas sinfónicas en Colombia, especie en vía de extinción. Agencia de Noticias UN. Recuperado de: http://agenciadenoticias.unal.edu.co/detalle/article/orquestas-sinfo nicas-en-colombia-especie-en-via-de-extincion.html

Ecos del Combeima. (2015). El gobernador Jaramillo acabó la Banda Sinfónica y ahora a los muchachos se les paga por concierto. Ecos del Combeima. Recuperado de: http://ecosdelcombeima.com/ibague/nota-53428-el-gobernador-jara millo-acabo-la-banda-sinfonica-y-ahora-a-los-muchachos-se-les-pa

Francel, A. (2017). Los edificios que pasaron la institución que queda. Gobernación del Tolima (Colombia) entre 1886 y 1957. HISTORelo, Revista de Historia Regional y Local, 9(17), 123-151. Recuperado de: http://www.scielo.org.co/pdf/histo/v9n17/2145-132X-histo-9-17-0 0123.pdf

Melo, A. (2007) Retrato de familia. Aquelarre, (12), 21 -26. Recuperado de: http://ccultural.ut.edu.co/images/Revistas\%20Aquelarre/Aquelarre \%2012.pdf

Muñoz, E. (2015). La música tradicional colombiana en los planes formativos del área instrumental y de conjuntos de la Licenciatura en Educación Musical de la Universidad Pedagógica Nacional. Trabajo de grado, Licenciatura en Música. Universidad Pedagógica Nacional. 
Ochoa, J. (2011). La práctica común como la menos común de las prácticas: una mirada critica a los supuestos que configuran la educación musical universitaria en Colombia. Tesis de Maestría. Pontificia Universidad Javeriana. Bogotá, Colombia.

Ospina, P. (2015). Panorama sobre el conocimiento construido acerca del jazz en los trabajos de grado de la Licenciatura en Música de la Universidad Pedagógica Nacional. Trabajo de grado, Licenciatura en Música. Universidad Pedagógica Nacional.

Pinar, W. (2004). La teoria del curriculum. Madrid, España: Narcea, S.A. de Ediciones.

Posner, G. (2005). Análisis del currículo. Traducción Miguel Sarmiento. México. Mcgraw-hill.

Prudencio, J. (2004) Entre olvidar y darse a olvidar: música para setenta gatos (mi recuerdo sobre Óscar Buenaventura). Aquelarre, 3(5), 55-62. Recuperado de: http://ccultural.ut.edu.co/images/Revistas\%20Aquelarr e/Aquelarre\%2005.pdf

Redacción El Tiempo. (2002). El desafine sinfónico nacional. El Tiempo. Recuperado de: https://www.eltiempo.com/archivo/documento/MAM1344777

Stake, S. (1999). Investigación con estudio de casos. Madrid, España: Ediciones Morata.

Zuleta, L. (2003). Impacto del sector fonográfico en la economía colombiana. Bogotá, Colombia: Edición del Convenio Andrés Bello. 